\title{
Méthodologie de l'évaluation des médicaments chez la femme enceinte
}

\author{
Marina Chauvenet, ${ }^{1}$ Alain Rimailho, ${ }^{2}$ Nathalie Hoog-Labouret ${ }^{1}$ et les participants à la table ronde \\ $n^{\circ} 5$ de Giens XVIII ${ }^{* *}$ \\ 1 Agence Française de Sécurité Sanitaire des Produits de Santé, Saint-Denis, France \\ 2 Schering-Plough, Levallois-Perret, France
}

\section{Résumé}

Objet de réticences diverses, la prescription médicamenteuse chez la femme enceinte répond pourtant à une absolue nécessité dans un certain nombre de cas. Une évaluation correcte du médicament s'impose donc, axée sur l'efficacité et la sécurité ou essentiellement sur la sécurité, selon l'expérience disponible en dehors de la grossesse. La méthodologie d'évaluation ne répond pas nécessairement à des caractéristiques particulières. Des recommandations de développement sont formulées suivant les situations les plus fréquentes ainsi que des propositions concrètes, pour permettre une meilleure sensibilisation des différents partenaires de santé impliqués (institutions, industriels et prescripteurs). En particulier, des incitations d'ordre réglementaire et économique, superposables à celles adoptées en Europe et aux Etats-Unis pour les maladies orphelines, devraient être mises en place pour favoriser l'évaluation obstétricale du médicament.

Mots clés : médicament, grossesse, évaluation, incitations réglementaires

Texte reçu le 6 mai 2003 ; accepté le 26 mai 2003

\section{Position du problème}

La prescription et la consommation médicamenteuses en cours de grossesse sont une réalité incontournable, à la fois non négligeable et diversifiée, puisqu'il a pu être établi que près de $35 \%$ de femmes prenaient au moins une fois un médicament, hormis les supplémentations en fer et en vitamines, pendant leur grossesse..$^{[1,2]}$

A ce jour, les mentions légales des médicaments susceptibles d'être prescrits pendant la grossesse sont en France ciblées systématiquement sur le risque médicamenteux vis-à-vis de l'enfant à naître, lequel regroupe trois types d'effets potentiels : tératogène (malformatif, lié aux expositions en début de grossesse), foetotoxique (avec un risque débutant au deuxième trimestre de la grossesse) et néonatal (le plus souvent lié à des expositions en fin de grossesse ou pendant l'accouchement).

L'évaluation fondamentale du risque médicamenteux chez la femme enceinte, qui conduit à la rédaction de ces mentions, avait fait l'objet d'une table ronde précédente. ${ }^{[3]}$ Elle n'a donc pas été retenue à nouveau dans le cadre plus global de la méthodologie de l'évaluation des médicaments chez la femme enceinte.

Le prescripteur potentiel de médicaments chez la femme enceinte trouve donc aujourd'hui des mentions d'information sur le risque pour l'enfant à naître, dans le paragraphe « Grossesse et Allaitement » de l'autorisation de mise sur le marché (AMM) de tout médicament ; mais il remarque dans le même temps l'extrême rareté des médicaments ayant une indication spécifique d'utilisation au cours de la grossesse, ainsi que l'absence quasi complète d'information sur la posologie à prescrire tout au long de son déroulement. Ceci est d'autant plus surprenant que l'on connaît les importantes modifications pharmacocinétiques et parfois dynamiques induites par les modifications physiologiques de la grossesse, notamment au troisième trimestre. ${ }^{[4]}$

On pourrait penser que cette situation est liée à une absence quasi totale d'évaluation des médicaments chez la femme enceinte, débouchant logiquement sur une impossibilité de proposer des règles d'utilisation validées.

Ainsi, alors que les nausées et / ou vomissements du premier

$\dagger$ Pour la liste des participants, voir en fin d'article.

* Les articles, analyses et propositions issus des Rencontres de Pharmacologie Clinique de Giens n'engagent que leurs auteurs et ne préjugent pas de la position de leur organisme de tutelle. 
trimestre de la grossesse sont fréquents et donnent lieu à un certain nombre de prescriptions d'antiémétiques, l'efficacité de ces derniers n'est pas clairement établie, notamment par rapport aux mesures non pharmacologiques. ${ }^{[5]}$ De même, bien que la spiramycine soit en France le traitement de référence institué à titre préventif pour réduire la transmission materno-fœtale en cas de séroconversion de toxoplasmose, sa réelle efficacité reste insuffisamment évaluée. ${ }^{[6]}$

Il est clair qu'un développement clinique chez la femme enceinte est délicat à envisager, de par la triple conjonction :

- du poids légitime des considérations éthiques au sein desquelles cependant se mêlent parfois, depuis l'affaire du thalidomide, certaines craintes irrationnelles ;

- de la frilosité des industriels liée aux problèmes médicolégaux et à la faiblesse de la reconnaissance économique ;

- et de l'absence d'obligation réglementaire à produire pour l'enregistrement des informations concernant l'utilisation chez la femme enceinte.

Il existe néanmoins des initiatives non négligeables démontrant l'intérêt et la faisabilité d'aborder de manière plus systématique la question de la méthodologie de l'évaluation des médicaments chez la femme enceinte.

On peut ainsi noter que la méthodologie Cochrane, fondée sur l'approche méta-analytique, a été appliquée à ses débuts à l'obstétrique pour laquelle nombre d'études médicamenteuses sont retrouvées. ${ }^{[7]}$

\section{Situation éthique et réglementaire}

Avant d'identifier les situations cliniques où un développement chez la femme enceinte apparait souhaitable, voire indispensable et de distinguer les outils disponibles, il convient de rappeler la situation actuelle, au plan éthique et réglementaire.

En France, la loi de protection des personnes qui se prêtent à des recherches biomédicales, dite " loi Huriet-Sérusclat », ${ }^{[8]}$ laisse la possibilité de réaliser des essais cliniques avec ou sans bénéfice individuel direct (BID) pendant la grossesse. Elle définit des conditions particulières de réalisation pour les recherches sans BID, lesquelles ne sauraient être entreprises que «si elles ne présentent aucun risque sérieux prévisible pour leur santé ou celle de leur enfant, si elles sont utiles à la connaissance des phénomènes de la grossesse ou de l'accouchement et qu'elles ne peuvent être réalisées autrement ». La nouvelle Directive Européenne ${ }^{[9]}$ sur les essais cliniques ne prévoit, quant à elle, aucune mention spécifique à la grossesse.

Toujours au plan national, l'information délivrée dans l'AMM dans la rubrique "Grossesse et Allaitement » résulte d'une évaluation de l'ensemble des données disponibles (précliniques et cliniques) par classes pharmacothérapeutiques sur le risque et le bénéfice en cours de grossesse, effectuée par un groupe de travail multidisciplinaire de la Commission d'AMM. Le but de cette démarche est d'aboutir à une conduite à tenir pratique pour le prescripteur, prenant en compte les besoins médicaux.

La réglementation européenne disponible concerne une obligation pour les industriels à déclarer les cas de grossesses rapportés lors d'une exposition à l'un de leurs médicaments, en particulier en cas d'anomalie dans le déroulement de la grossesse ou pendant la période néonatale et $a$ fortiori en cas de malformation. ${ }^{[10]}$ Mais l'évaluation du médicament administré pendant la grossesse semble se structurer davantage au niveau européen : des recommandations (ou «guidelines ») destinées à renforcer les obligations de suivi post-AMM pour les grossesses exposées à une liste de médicaments jugés prioritaires, sont en cours d'élaboration et un groupe de travail chargé d'harmoniser les modalités d'évaluation et d'information sur la grossesse dans le Résumé des Caractéristiques du Produit, a été mis en place.

Enfin, il faut mentionner que la «US Food and Drug Administration » a débuté une réflexion sur l'évaluation pharmacoclinique des médicaments administrés aux deuxième et troisième trimestres de la grossesse et sur les exigences requises concernant les registres chez la femme enceinte.

Il apparaît clairement que des essais cliniques peuvent tout à fait s'inscrire dans le plan de développement d'un médicament en incluant des femmes enceintes.

En France, 25 essais cliniques portant sur l'évaluation d'un médicament chez la femme enceinte ont été déclarés à l'Afssaps (Agence française de sécurité sanitaire des produits de santé) pour la période s'échelonnant de janvier 1998 à septembre 2002. Ces essais étaient majoritairement des essais avec BID. Leur promoteur était un industriel dans seulement neuf cas.

Ceci doit bien entendu être mis en parallèle avec les 1500 essais cliniques environ, toutes populations confondues, déclarés annuellement, dont une centaine concerne l'enfant.

Il a été jugé utile de procéder à l'analyse d'un passé récent pour identifier à partir de quels éléments il avait été ou il serait possible de proposer des mentions légales pour des produits développés ou utilisés au cours de la grossesse. L'exemple de l'atosiban récemment enregistré en procédure européenne centralisée dans une indication purement obstétricale, après un développement complet, est en cela exemplaire. A l'opposé, dans la même indication (menace d'accouchement prématuré [MAP]), la nifédipine fait l'objet d'un usage certain hors AMM, fondé sur une démonstration d'efficacité admise versus $\beta$-mimétiques associée à une meilleure tolérance, ${ }^{[11]}$ alors que persistent des incertitudes sur la sécurité néonatale par rapport à des effets rares 
mais potentiellement graves. ${ }^{[12]}$ Par ailleurs, l'Afssaps a mené, en concertation avec les industriels, une démarche d'évaluation fondée sur l'appréciation du risque fotal par l'étude du passage transplacentaire, pour permettre d'alléger les mentions légales de certaines héparines de bas poids moléculaire, en fonction des besoins médicaux.

\section{Pharmacocinétique mère / enfant}

Il est fondamental de disposer d'informations pertinentes sur la pharmacocinétique du médicament, notamment sur son degré de passage transplacentaire.

La grossesse, en effet, modifie les paramètres pharmacocinétiques en augmentant la durée de la vidange gastrique, en ralentissant le transit intestinal, en augmentant la volémie et en augmentant la filtration glomérulaire. Ces modifications peuvent affecter l'absorption, la biodisponibilité et l'élimination de nombreux médicaments.

Le passage transplacentaire procède surtout d'une diffusion passive, en fonction des caractéristiques moléculaires du médicament (liposolubilité, masse moléculaire, $\mathrm{pH}$, degré de fixation protéique, activité enzymatique propre placentaire). ${ }^{[13]}$

En pratique, il peut être considéré que, à l'exception de molécules de gros poids moléculaire type insuline, la quasitotalité des médicaments passe le placenta. Il convient donc, pour tenter de cerner l'importance du passage transplacentaire d'un médicament, de recourir à l'analyse d'éléments venant de modèles animaux, de données humaines ex vivo (technique du placenta perfusé) et de données humaines in vivo comparant les taux circulants mère-fœtus avec sang prélevé au cordon à la naissance.

Cependant chacune de ces méthodes n'offre qu'une vision réduite de la situation physiologique. Les dosages au cordon à la naissance ne sont qu'un instantané ne tenant pas compte de la cinétique ; leur interprétation nécessite un nombre relativement important de prélèvements chez des couples mère-cordon. Les techniques ex vivo offrent une approche dynamique, mais sont cependant techniquement délicates à mettre en œuvre ; elles nécessitent donc une expertise particulière et des contrôles de qualité. Elles peuvent surtout être utiles pour comparer, au sein d'une même classe de produits, les passages placentaires différenciés. ${ }^{[14]}$ Pour les classes de médicaments dont le passage placentaire se fait par simple diffusion, les résultats in vivo et ex vivo sont concordants. ${ }^{[15]}$ A l'inverse, on peut citer des exemples où les deux approches ont montré des résultats discordants, tels qu'avec des antirétroviraux de la classe des inhibiteurs de protéase, pour lesquels les études initiales in vivo suggéraient une absence de passage placentaire, ${ }^{[16]}$ alors que les études ex vivo ont démontré des différences de passage selon la molécule. ${ }^{[17,18]}$

C'est de la confrontation de l'ensemble des données théoriques propres au médicament, animales et humaines obtenues lors des phases précoces de développement (en particulier d'ordre pharmacocinétique) que naît l'estimation qualifiée plus que quantifiée précisément, des marges thérapeutiques à attendre du développement clinique d'un nouveau produit, pour la mère comme pour le fotus. En disposant ainsi d'un rapport des concentrations sanguines fœto-maternelles (à la naissance), d'un profil pharmacocinétique chez la mère et de données obtenues ex vivo, on peut considérer disposer de suffisamment d'informations à titre de pré-requis pour s'engager dans l'évaluation clinique large du produit. Ceci vient s'ajouter aux exigences de la directive ICH (International Conference on Harmonisation) de 1997 qui indique qu'avant d'engager des femmes enceintes dans un essai clinique, toutes les études de reproduction et la batterie standard des tests de génotoxicité devraient être conduites. ${ }^{[19]}$

\section{Les différentes situations pathologiques}

Quatre situations pathologiques différentes peuvent être reconnues :

1. Les pathologies exclusivement fœtales, dont l'incidence est faible et que nous exclurons du champ de la réflexion (exemples : spiramycine et sulfadiazine-pyriméthamine dans la toxoplasmose, anti-arythmiques dans les tachycardies supraventriculaires fœtales, L-thyroxine dans les hypothyrö̈dies in utero, etc...).

2. Les pathologies gravidiques pures pour lesquelles il n'existe, par définition, aucune indication en dehors de la grossesse. Il s'agit principalement des médicaments visant à déclencher le travail ou à l'inverse les tocolytiques (exemple de l'atosiban).

Une fois constitués les pré-requis plus haut mentionnés, il est possible de planifier un ou des essais cliniques, randomisés versus placebo ou produit de référence s'il existe, visant à démontrer l'efficacité. De tels essais ne peuvent, par définition, être menés que chez des femmes enceintes. Le choix du critère principal peut être dicté par le souci de réduire le nombre de patientes nécessaires, imposant alors une réflexion sur la validité du critère de substitution alors éventuellement retenu, surtout si une phase de recherche de dose apparaît incontournable avant de procéder à l'étude proposée de phase III.

Il convient aussi de prévoir, puis d'organiser le suivi du devenir des enfants de manière systématique, et ceci bien au-delà de la période néonatale. 
Au terme de ce développement, une pleine indication obstétricale doit pouvoir être accordée au produit étudié, si un rapport bénéfice / risque positif est établi sur des effectifs suffisants pour l'analyse d'efficacité et de sécurité (mère, enfant).

3. Les pathologies gravidiques pour lesquelles un médicament déjà testé et validé chez l'homme semble capable d'apporter le même bénéfice à la femme enceinte et peut-être aussi à son fœtus comme dans les maladies infectieuses transmissibles, par exemple le SIDA ou l'herpès. ${ }^{[20,21]}$ Il convient ici d'apprécier le plus tôt possible le juste besoin médical de la mère et du foetus, c'est-à-dire de savoir prendre le risque d'engager des essais cliniques visant à démontrer l'efficacité tôt dans la vie du produit, ce qui a été le cas dans la situation d'extrême urgence rencontrée lors de l'épidémie de SIDA avec un taux de transmission materno-foetale de $20-25 \%$. Ceci est possible dès que le prérequis pharmacocinétique plus haut décrit est établi, associé au recueil de pharmacovigilance spontanée incluant les cas signalés de grossesse survenant lors de la prescription du produit étudié, ainsi qu'aux données issues d'éventuelles cohortes. Un essai clinique classique visant à démontrer l'efficacité peut être engagé, comparatif, randomisé versus produit de référence ou versus placebo si la référence n'existe pas ou est insuffisamment évaluée chez la femme enceinte dans cette situation. Une réflexion, ici aussi, sur l'intérêt et la validité d'un critère de substitution peut s'avérer nécessaire s'il apparaît que les effectifs à inclure sont rendus trop importants par le choix du critère principal retenu et par l'incidence faible de l'événement à contrôler par le produit.

Il faudrait normalement prévoir l'organisation du suivi du devenir des enfants, mais ceci pose des problèmes majeurs de faisabilité, notamment à cause du coût engendré.

Au terme de ce développement, une indication spécifique du produit chez la femme enceinte devrait être obtenue, si un rapport bénéfice / risque favorable a été établi.

Ainsi, si dans les situations deux et trois ci-dessus la recherche d'une démonstration d'efficacité sera exigée en plus de recueil de sécurité et pourra dans le meilleur des cas aboutir à une indication obstétricale, dans la situation quatre présentée ci-après l'évaluation de la sécurité sera prépondérante et ses conclusions pourront se traduire par une modification appropriée de l'information sur le médicament (conduite à tenir).

4. Les pathologies chroniques préexistantes à la grossesse et dont le traitement doit être poursuivi pendant la grossesse (par exemple : épilepsie, asthme ou dépression).

Il faut ici poser les deux questions suivantes :

- la grossesse modifie-t-elle la physiopathologie et donc peutêtre le profil évolutif de la maladie ?
- la grossesse modifie-t-elle les caractéristiques pharmacologiques d'un médicament donné ?

Répondre négativement à ces deux questions sur des arguments raisonnés devrait permettre, en s'appuyant sur les données actualisées de pharmacovigilance, de pouvoir introduire dans les mentions légales du médicament une information et donc une orientation pour le prescripteur, allant jusqu'à des considérations de dose en cas d'utilisation pendant la grossesse.

Inversement, répondre positivement à l'une de ces questions devrait inciter à réaliser un profil pharmacocinétique du produit concerné, au moins au cours du troisième trimestre de la grossesse, pour permettre ici encore avec les données actualisées de la pharmacovigilance, d'introduire dans les mentions légales une information d'orientation posologique pour le prescripteur. Ceci lèverait le souci éthique fréquemment relevé, ${ }^{[22]}$ découlant d'une absence complète de mention d'orientation posologique d'un médicament durant la grossesse. En effet, les modifications physiologiques peuvent induire de profondes modifications pharmacocinétiques du médicament et rendre ainsi inefficace un produit aux concentrations très abaissées pendant plusieurs semaines ou mois, alors que la posologie usuelle aura été maintenue pendant la grossesse. ${ }^{[23]}$ Cette étude pharmacocinétique serait d'autant plus indiquée que le médicament a une marge thérapeutique étroite et qu'une corrélation a pu être établie, en dehors de la grossesse, entre la dose et les concentrations plasmatiques d'une part, et la dose et l'effet pharmacologique, d'autre part.

\section{L'approche pragmatique}

Au-delà de cette approche classique, la possibilité d'adopter une attitude plus pragmatique dans certains cas a été discutée. En effet, plusieurs molécules ont fait l'objet d'un ou plusieurs essais d'efficacité, sans qu'il y ait eu d'étude préalable de phase I / II. Parfois, les essais ont été d'une qualité suffisante pour être regroupés dans le cadre de méta-analyses (exemple des antagonistes calciques dans le traitement des MAP ou du misoprostol dans le déclenchement du travail). De plus, des informations parfois conséquentes en matière de pharmacovigilance et donc de sécurité d'utilisation sont disponibles au travers de registres de pharmacovigilance (exemple de l'aciclovir).

Ne pourrait-on point, surtout lorsque les pratiques se trouvent éventuellement validées par des recommandations émises lors de conférences de consensus, envisager une validation réglementaire, permettant de déboucher sur une modification sensible de l'information délivrée au prescripteur ? Cette proposition concerne des produits souvent anciens et donc génériqués de longue date, pour lesquels il serait en pratique très difficile 
Tableau I. Proposition de spécialités fréquemment utilisées en pratique obstétricale

\begin{tabular}{ll}
\hline Produit (DCI) & Utilisation \\
\hline Nifédipine & MAP \\
Nicardipine & MAP \\
Misoprostol & Déclenchement du travail \\
Aciclovir & Prévention récurrence herpès génital \\
Amoxicilline & Rupture prématurée des membranes \\
Acide chénodésoxycholique & Cholestase gravidique \\
Trinitrine & Tocolyse en urgence \\
\hline
\end{tabular}

$\overline{\mathbf{D C l}}=$ dénomination commune internationale ; MAP = menace d'accouchement prématuré.

d'envisager d'intéresser les industriels à des plans de développement spécifiques à la femme enceinte. Une liste établie par la table ronde est proposée à titre indicatif (tableau I).

\section{Les points pour action}

\section{1 Sur le plan politique}

Le rôle des institutions apparaît crucial pour véritablement structurer et développer l'évaluation du médicament en obstétrique.

\subsubsection{Propositions européennes d'ordre réglementaire}

La proposition d'appliquer l'approche de « maladie orpheline » aux pathologies spécifiquement gravidiques, pour lesquelles certains produits ne revendiqueraient que cette indication, a fait l'unanimité. Cette disposition serait destinée aux industriels et peut-être aussi aux institutionnels à même de promouvoir des essais cliniques. Des incitations d'ordre réglementaire et économique, superposables à celles adoptées en Europe $^{[24]}$ et aux Etats-Unis ${ }^{[25]}$ pour les médicaments orphelins, seraient seules à même de dynamiser un courant de recherche et surtout de développement spécifique à la femme enceinte. De même, compte tenu de l'intrication inévitable entre le statut du fœus et celui du nouveau-né, né à terme ou a fortiori prématuré, il semble légitime que la situation de la femme enceinte soit au moins envisagée dans le futur règlement pédiatrique européen.

\subsubsection{Proposition nationale d'ordre épidémiologique}

Les cliniciens et épidémiologistes du groupe estiment nécessaire qu'après des années de «gestation » et une expérience départementale réussie, ${ }^{[26]}$ s' organise enfin en France un registre national d'évolution des grossesses recensées ou « certificat d'issue de grossesse », dans lequel un certain nombre d'informations pourraient être analysées, en particulier la notion d'exposition médicamenteuse avec, par exemple, un recueil systématique de certaines expositions pendant des périodes limitées. A défaut, l'intérêt d'informatiser le dossier obstétrical à l'échelon de différentes régions a été souligné.

6.2 Sur le plan réglementaire

\subsection{Information délivrée par l'autorisation de mise sur le marché}

A l'initiative de l'Afssaps, il a été envisagé de façon pragmatique la possibilité de réviser rapidement l'information de la rubrique Grossesse d'une liste de spécialités jugées « prioritaires » en termes de besoin obstétrical et ayant fait l'objet d'une «certaine » évaluation validée par la littérature. Cette liste (tableau I), proposée par les obstétriciens de la table ronde, nécessiterait d'être discutée en concertation avec les sociétés savantes.

Similaire aux initiatives de l'Afssaps menées dans le domaine du médicament pédiatrique, cette démarche aurait pour objectif de réduire l'écart encore considérable entre l'information de l'AMM et les pratiques, au moins pour les médicaments jugés «de première nécessité ». A ce propos, la problématique similaire mais non identique de l'allaitement maternel mériterait également plus d'attention. L'information des spécialités qui aurait été validée selon la grille actuelle (juillet 2002) du groupe Ad Hoc de l'Afssaps mériterait un «étoilage » au niveau du Dictionnaire des Spécialités Vidal pour offrir une meilleure lisibilité au prescripteur, en particulier eu égard aux dysharmonies de libellé induites par les procédures européennes d'enregistrement, par rapport à l'évaluation nationale. Cette information devrait également être disponible sur le site Internet de l'Afssaps.

Dans le même esprit d'harmonisation, l'Afssaps devrait s'impliquer particulièrement dans l'élaboration des différents prochains « guidelines » européens, pour faire valoir l'expérience française.

\subsubsection{Révision de la loi Huriet-Sérusclat}

Dans la perspective d'une transposition en droit national de la nouvelle Directive Européenne sur les essais cliniques, il y aurait lieu a minima de maintenir la distinction actuelle entre les essais avec ou sans BID. Le groupe propose également qu'à l'instar des besoins exprimés pour le médicament pédiatrique, figure une mention particulière concernant les compétences requises en obstétrique et néonatologie auprès des Comités Consultatifs de Protection des Personnes dans la Recherche Biomédicale (CCPPRB), qui seraient amenés à examiner le bien-fondé de protocoles obstétricaux. 


\subsection{Sur le plan éthique}

Une réflexion mériterait d'être menée, en liaison avec le Comité National d'Ethique et diverses personnalités, car les réticences les plus fortes ne se trouvent pas nécessairement au niveau de la femme elle-même et de son couple, ni des cliniciens, mais aussi au niveau de l'industrie, voire des institutionnels.

\section{Conclusion}

Le développement clinique des médicaments pendant la grossesse se heurte à des difficultés de tous ordres bien identifiées, notamment liées à l'absence d'obligation industrielle de fournir des données chez la femme enceinte pour l'enregistrement, sauf évidemment en cas d'indication obstétricale visée. Pour réellement faire progresser la connaissance et l'usage du médicament chez la femme enceinte, une véritable volonté politique nationale et européenne apparaît nécessaire. Celle-ci impliquerait principalement d'intégrer dans le récent règlement européen sur les médicaments orphelins, les médicaments visant le traitement de pathologies purement obstétricales et de mettre en place en France un registre des grossesses, à l'instar de ce qui existe déjà en Suède par exemple. Par ailleurs, il semble indispensable de mieux cerner les besoins médicaux prioritaires et de proposer en conséquence, en fonction des données disponibles, l'information la mieux adaptée pour le prescripteur dans l'AMM. Ces propositions devraient améliorer une situation globalement considérée à l'heure actuelle comme peu satisfaisante car, si l'évaluation légitime du risque pour l'enfant à naître doit rester au cœur des préoccupations, elle ne doit pas occulter la nécessité d'éclaircir les conditions d'une évaluation raisonnée du bénéfice et du risque pour la mère et du bénéfice pour l'enfant.

\section{Participants}

F. Audibert (Hôpital Antoine Béclère, Clamart), F. Bavoux (Hôpital Saint-Vincent de Paul, Paris), M.J. Boutroy (CHU, Nancy), D. Brasseur (EMEA, CPMP, Londres), P. Carlier (Hôpital Fernand Widal, Paris), E. Elefant (CHU Saint-Antoine, CRAT, Paris), M. Gersberg (Sanofi-Synthelabo, Le Plessis-Robinson), F. Goffinet (Maternité Port-Royal, Paris), E. JacqzAigrain (Hôpital Robert Debré, Paris), D. Lemery (Maternité de l'Hôtel-Dieu, Clermont-Ferrand), L. Mandelbrot (CHU Cochin, Paris), V. Mirelesse (Institut de Puériculture et Périnatalogie, Paris), B. Saint-Salvi (Afssaps, StDenis), C. Tchinou (Afssaps, St-Denis), T. Vial (Centre Hospitalier E. Herriot, Lyon).

\section{Références}

1. Lacroix I, Damase-Michel M, Lapeyre-Mestre M, et al. Prescription of drugs during pregnancy in France. Lancet 2000; 356 (9243): 1735-6

2. McElhatton P. Pregnant women and drug safety. Good Clin Pract J 2002; 9: 15-7

3. Eléfant E, Sainte-Croix A, et les membres de la table ronde $\mathrm{n}^{\circ} 6$ de Giens XII. Evaluation du risque médicamenteux chez la femme enceinte : méthodologie d'évaluation et gestion du risque. Thérapie 1997; 52: 307-11
4. Loebstein R, Lalkin A, Koren G. Pharmacokinetic changes during pregnancy and their clinical relevance. Clin Pharmacokinet 1997; 33: 328-43

5. Mazzotta P, Magee LA. A risk-benefit assessment of pharmacological and nonpharmacological treatments for nausea and vomiting of pregnancy. Drugs 2000; 59: 781-800

6. Wallon M, Liou C, Peyron F. Congenital toxoplasmosis: systematic review of evidence of efficacy of treatment in pregnancy. BMJ 1999; 318: 1511-4

7. Anonymous. Stratégies : menaces d'accouchement prématuré. Prescrire 2002; 22: 676-686

8. Loi pour la protection des personnes qui se prêtent à des recherches biomédicales. Code de la Santé Publique. Livre II bis : article L209-4

9. Directive 2001/20/CE du Parlement européen et du Conseil du 4 avril 2001 concernant le rapprochement des dispositions législatives, réglementaires et administratives des États membres relatives à l'application de bonnes pratiques cliniques dans la conduite d'essais cliniques de médicaments à usage humain [online]. Journal Officiel des Communités Europeannes n ${ }^{\circ}$ L121 -1/05/2001. Available from URL: http://europa.eu.int/eur-lex/fr/archive/2001/ 1_12120010501fr.html

10. European Agency for the Evaluation of Medicinal Products (EMEA). Notice to marketing authorization holders: pharmacovigilance guidelines [online]. CPMP/PhVWP/108/99, 1999 Jan 29. Available from URL: http://www.tga. health.gov.au/docs/pdf/euguide/phvwp/010899en.pdf

11. Tsatsaris V, Papatsonis D, Goffinet F, et al. Tocolysis with nifedipine or beta-adrenergic agonists: a meta-analysis. Obstet Gynecol 2001; 97: 840-7

12. Rosen LJ. The great tocolytic debate: some pitfalls in the study of safety. Am J Obstet Gynecol 2001; 184: 1-7

13. Gablot D, Laurichesse H, Lemery D. Prévention des risques fæataux. Rev Prat 2002; 52: 751-64

14. Bourget P, Roulot C, Fernandez H. Transfert transplacentaire et métabolisme fotoplacentaire des médicaments : méthodes d'études, apports et implications thérapeutiques. Thérapie 1994; 49: 481-97

15. Tuntland T, Odinecs A, Pereira CM, et al. In vitro models to predict the in vivo mechanism, rate, and extent of placental transfer of dideoxynucleoside drugs against human immunodeficiency virus. Am J Obstet Gynecol 1999; 180: 198206

16. Mirochnick M, Dorenbaum A, Holland D, et al. Concentrations of protease inhibitors in cord blood after in utero exposure. Pediatr Infect Dis J 2002; 21: 835-8

17. Forestier F, de Renty P, Peytavin G, et al. Maternal-fetal transfer of saquinavir studied in the ex vivo placental perfusion model. Am J Obstet Gynecol 2001; 185: $178-81$

18. Casey BM, Bawdon RE. Placental transfer of ritonavir with zidovudine in the ex vivo placental perfusion model. Am J Obstet Gynecol 1998; 179: 758-61

19. International Conference on Harmonisation of Technical Requirements for Registration of Pharmaceuticals for Human Use (ICH). Therapeutic Products Directorate Guideline ICH. Harmonized tripartite guideline (M3): timing of non-clinical safety studies for the conduct of human clinical trials for pharmaceuticals, 1997 Jul

20. Mandelbrot L. Syndrome d'immunodéficience acquise et grossesse. Encycl Méd Chir (Traité d'Obstétrique) 2001: 5-039-D-40

21. Mandelbrot L. Vertical transmissions of viral infections. Curr Opin Obstet Gynecol 1998; 10: 123-8

22. Wood SF, Miller MA, Hollinger KA. Why women should be included in clinical trials? Good Clin Pract J 2002 Sep; 9: 3-4

23. Gérard-Coué MJ, Bréart G, Spriet A. Les femmes dans les essais cliniques (Giens X). Thérapie 1995; 50: 363-8

24. Règlement 141/2000 du parlement européen et du conseil du 16 décembre. Journal Officiel des Communités Européennes 2000 ; nº 018 du 22 janvier 2000 : 0001-0005

25. Orphan drug regulations: final rule. Federal Register 62076 V.57, 1992

26. Papiernik E, Bucourt M, Zeitlin J, et al. Evolution de la régionalisation des soins périnatals dans le département de la Seine-Saint-Denis de 1989 à 1999. J Gynécol Obstét Biol Reprod (Paris) 2001; 30: 338-43

Correspondance et offprints : Alain Rimailho, Schering-Plough, 92 rue Baudin, 92307 Levallois-Perret Cedex, France.

E-mail : alain.rimailho@spcorp.com 